\title{
Per Diem Payment Method
}

National Cancer Institute

\section{Source}

National Cancer Institute. Per Diem Payment Method. NCI Thesaurus. Code C70681.

The payment mechanism that is used to compensate a healthcare provider for the care provided to a patient in form of a fee for each day of care, covering all services rendered during this day. 